Chinese Journal of Organic Chemistry

\title{
含噻唑骨架的吲哚衍生物的有效合成
}

\author{
高文涛* 兰帅郑宏梅白仁青卓玛
}

(渤海大学超精细化学品研究所 锦州 121000)

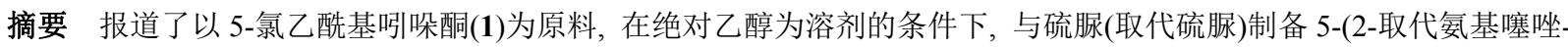
4-基)二氢吲哚-2-酮类化合物 $\mathbf{2 a} \sim \mathbf{2 k}$ ，与硫代酰胺合成 5-(2-取代噻唑-4-基)二氢吲哚-2-酮类化合物 21 $\sim 2 \mathbf{2}$. 该反应操作 简便、条件温和、收率良好. 所有化合物未见文献报道, 其结构经红外光谱、质谱、核磁氢谱(碳谱)及元素分析确认. 关键词 5-氯乙酰基吲哚酮; 硫嫝; 硫代酰胺; 噻唑; 吲哚衍生物; 合成
\end{abstract}

\section{A Facile Synthesis of Thiazole Skeleton-Containing Indole Derivatives}

\author{
Gao, Wentao* Lan, Shuai Zheng, Hongmei Bairenqing, Zhuoma \\ (Institute of Superfine Chemicals, Bohai University, Jinzhou 121000)
}

\begin{abstract}
An efficient and easy method for the synthesis of thiazole-containing indole derivatives was described, involving the condensation reaction of 5-chloroacetyloxindole with thiourea (substituted thiourea) and thioamide in the solvent of absolute ethanol via Hantzseh reaction to give the corresponding 5-(2-substituted-thiazol-4-yl)indolin-2-one $(\mathbf{2 a} \sim \mathbf{2 k})$ and 5-(2-substituted-thiazol-4-yl)indolin-2-one (2I $\sim \mathbf{2 q})$, respectively. The procedure has the advantages of simple operation, mild reaction conditions, and good yields. All of compounds were new and their structures were confirmed by IR, MS, ${ }^{1} \mathrm{H}$ NMR and ${ }^{13} \mathrm{C}$ NMR spectra and elemental analyses.
\end{abstract}

Keywords 5-chloroacetyloxindole; thioamide; thiourea; thiazole-containing; indole derivatives; synthesis

噻唑是一类含氮硫杂原子的五元芳杂环，这种结构 赋予了噻唑类化合物诸多特殊的性能，在众多领域具有 广泛的潜在应用, 引起化学工作者和药学家的极大关 注 $^{[1,2]}$. 在医药领域, 噻唑类化合物表现出抗细菌 ${ }^{[3]}$ 、抗 病毒 ${ }^{[4]}$ 、抗癌 ${ }^{[3]}$ 、抗结核 ${ }^{[6]}$ 、消炎镇痛 ${ }^{[7]}$ 和抗寄生虫 ${ }^{[8]}$ 等 多种生物活性; 在农业领域, 噻唑类化合物作为良好的 除草剂 ${ }^{[9]}$ 、植物生长调节剂 ${ }^{[10]}$ 以及杀菌和杀虫剂 ${ }^{[11 \sim 13]}$ 得到广泛应用, 如杀菌剂噻唑菌胺, 噻菌灵、噻唑禾草 灵, 除草剂苯噻草胺等; 在材料领域, 由于噻唑分子内 含富电子氮硫杂原子及共轭体系, 表现出许多独特的性 能, 在新兴有机材料领域有着重要的应用 ${ }^{[14,15]}$.

吲哚类衍生物是广泛存在于自然界的重要杂环化 合物, 因其具有独特的化学和生理药理活性, 引起人们

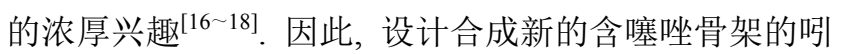
哚衍生物用于各种药物开发并且与其它吲哚衍生物进
行比较具有重要的理论和现实意义. 为此我们以 5 -氯乙 酰基吲哚酮为底物，与硫脲(取代硫艮)、硫代酰胺在绝 对乙醇中回流反应, 得到一系列具有多个活性基团的含 噻唑骨架的吲哚类化合物 $\mathbf{2 a} \sim \mathbf{2 q}$. 该系列化合物迄今 未见文献报道, 合成路线见 Scheme 1.

\section{1 结果与讨论}

\subsection{5-(2-取代氨基噻唑-4-基)二氢吲哚-2-酮类化合物} 的合成

形成噻唑环的方法有很多, 如 Hantzsch 等 ${ }^{[19]}$ 用 $\alpha$ 卤代酮和硫代酰胺或硫嫝反应，成功合成了三取代噻唑 (硫氮杂茂); Narender 等 ${ }^{[20]}$ 以 $\alpha$-溴代芳酮和硫脲(取代 硫脲)在 $\beta$-环糊精催化下合成了噻唑衍生物; Das 等 ${ }^{\left[{ }^{[1]}\right.}$ 以 $\alpha$-溴代芳酮和硫腿以及取代硫脲为原料, 在磷钿酸铵 (AMP)催化下合成了 4-芳基-2-氨基噻唑类化合物; Ro-

*E-mail: isfc@bhu.edu.cn

Received July 10, 2014; revised September 3, 2014; published online September 25, 2014.

Project supported by the National Natural Science Foundation of China (No. 21476028) and the Natural Science Foundation of Liaoning Province (No. 20120201).

国家自然科学基金(No. 21476028)和辽宁省自然科学基金(No. 20120201)资助项目. 
<smiles>[R]Nc1nc(-c2ccc3c(c2)CC(=O)N3)cs1</smiles>

$2 \mathbf{a} \sim 2 \mathrm{k}$

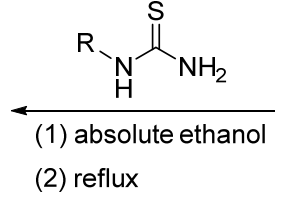

(3) $\mathrm{CH}_{3} \mathrm{COONa}$<smiles>O=C1Cc2cc(C(=O)CCl)ccc2N1</smiles>

1

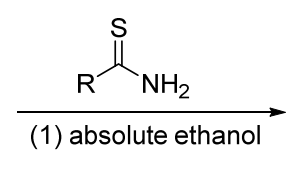

(2) reflux

(3) $\mathrm{CH}_{3} \mathrm{COONa}$<smiles>[R]c1nc(-c2ccc3c(c2)CC(=O)N3)cs1</smiles>

2I $\sim 2 q$

图式 1 含噻唑骨架的吲哚衍生物 $\mathbf{2 a} \sim \mathbf{2 q}$ 的合成

Scheme 1 Synthetic route of thiazole containing indole derivatives $\mathbf{2 a} \sim \mathbf{2 q}$

stamizadeh 等 ${ }^{[22]}$ 以 $\alpha$-溴代芳酮和硫艮(取代硫䐂)为原料, 以水为溶剂, 硫酸氢钠为催化剂, 得到了 2-氨基噻唑类 化合物; 2010 年 Jawale 等 ${ }^{[23]}$ 在常温下以 PEG-400 为介 质, 一锅法合成 2-氨基噻唑类化合物. 我们主要采用 Hantzseh 法形成噻唑环, 通过参考不同文献合成噻唑环 过程中的溶剂, 同时考虑到在反应过程中溶剂对底物的 溶解性以及溶剂的极性, 为此, 我们以 5-氯乙酰基吲梠 酮为底物与苯基硫脲反应, 考察了不同溶剂在回流的条 件下的反应产率, 结果如表 1 .

由于水对此反应有一定的影响, 为此以上所有溶剂 均通过干燥脱水处理. 通过对比试验我们发现反应中 $\mathrm{CS}_{2}$ 作为溶剂溶解效果最好, 但是其毒性过大, 且易挥 发, 对反应的控制起到了阻碍的作用且收率一般; 采用 无溶剂研磨的方法, 反应没有进行; 由于此反应为亲核
加成反应, 对溶剂极性的要求较高, 为此我们分别尝试 使用乙醇、丙醇、丁醇为溶剂, 试验结果表明绝对乙醇 和丙醇收率相似, 丁醇略低, 为此我们选用干燥脱水处 理的绝对乙醇为反应溶剂.

我们进行了拓展实验, 用绝对乙醇为溶剂, 5-氯乙 酰基吲哚酮为原料，与其它取代硫艮在回流的条件下合 成了吲哚酮联噻唑类化合物 $\mathbf{2 a} \sim \mathbf{2 k}$, 改进后的反应见 Scheme 2, 反应结果见表 2.

从表中数据可以看出, 5-氯乙酰基吲哚酮与取代硫 脲在绝对乙醇中表现出较高的反应活性，以及良好的收 率(74\% 89\%). 同时，我们发现取代基的空间位阻对 反应时间是有影响的，底物与氨基硫腿反应完成时间为 $6 \mathrm{~h}$ ，随着取代基团空间位阻的增加，反应时间也相对增 加，但空间位阻对反应的产率基本没有影响.

表 1 溶剂对反应产率的影响

Table 1 Effect of solvent on the yield

\begin{tabular}{lcccccccc}
\hline Entry & 1 & 2 & 3 & 4 & 5 & 6 & 7 \\
\hline Solution & $\mathrm{EtOH}$ & $\mathrm{CH}_{3} \mathrm{CN}$ & $\mathrm{CS}_{2}$ & $\mathrm{THF}$ & $\mathrm{CH}_{3}\left(\mathrm{CH}_{2}\right)_{2} \mathrm{OH}$ & $\mathrm{CH}_{3}\left(\mathrm{CH}_{2}\right)_{3} \mathrm{OH}$ & $\mathrm{No} \mathrm{solution}^{2}$ \\
Yield $^{a} \%$ & 89 & 61 & 76 & 80 & 88 & 84 & SM \\
\hline
\end{tabular}

${ }^{a}$ 分离产率.<smiles>O=C1Cc2cc(C(=O)CCl)ccc2N1</smiles><smiles>[R]NC(N)=S</smiles>

(2) reflux

(3) $\mathrm{CH}_{3} \mathrm{COONa}$<smiles>[Z20]Cc1ccc2c(c1)CC(=O)N2</smiles>

2a $\sim 2 k$

图式 2 5-(2-取代氨基噻唑-4-基)二氢吲哚-2-酮类化合物的合成

Scheme 2 Synthetic route of 5-(2-substituted-aminothiazol-4-yl)indolin-2-one derivatives

表 25 -氯乙酰基吲哚酮与硫脲(取代硫艮)反应结果

Table 2 Reaction results of 5-chloroacetyloxindole and thiourea (substituted thiourea)

\begin{tabular}{|c|c|c|c|c|}
\hline Entry & Compd. & m.p. $/{ }^{\circ} \mathrm{C}$ & Time/h & Yield $^{a} / \%$ \\
\hline 1 & & $278.9 \sim 280.2$ & 6 & 82 \\
\hline
\end{tabular}




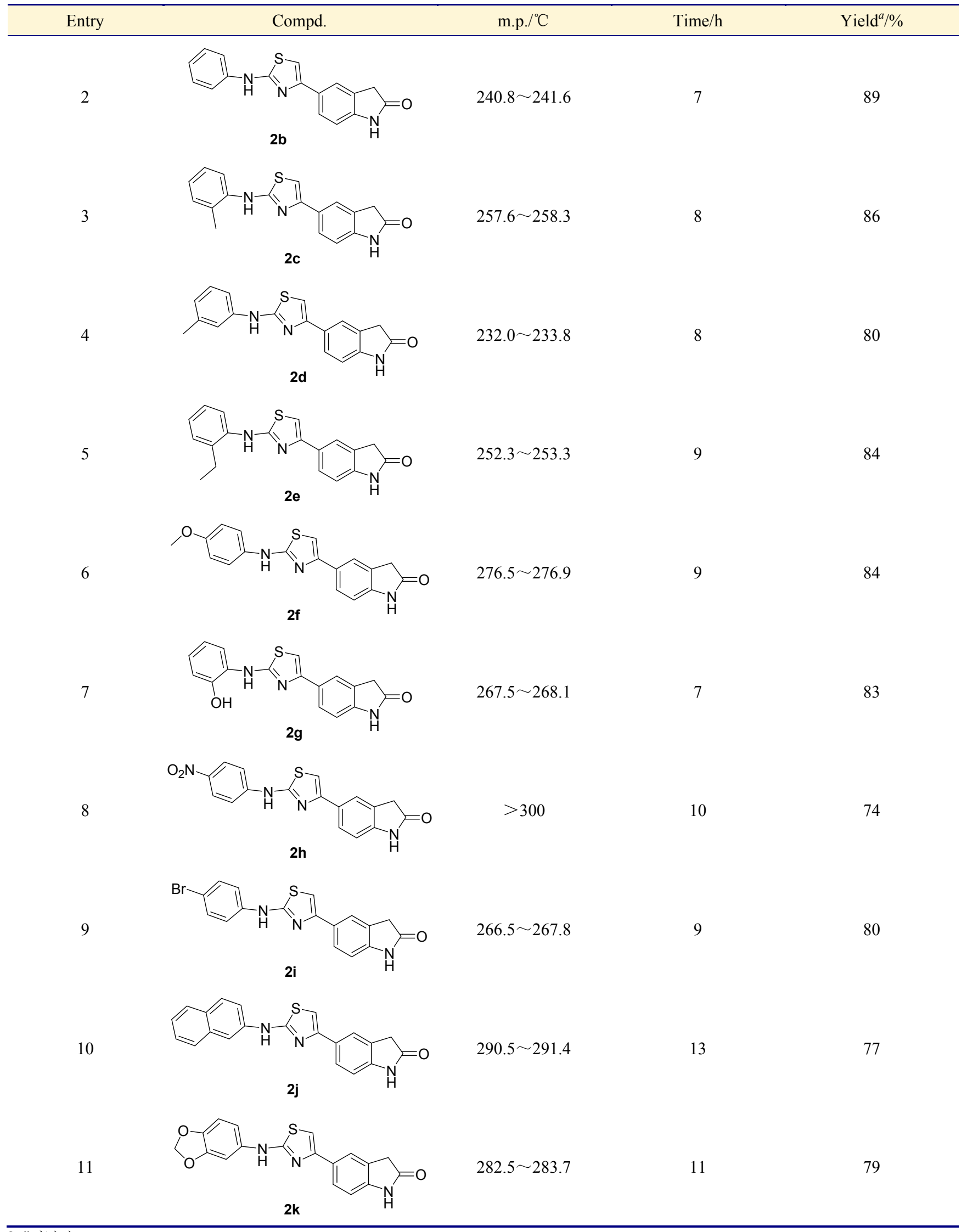

$a$ 分离产率. 


\subsection{5-(2-取代噻唑-4-基)二氢吲哚-2-酮类化合物的合} 成

以 5-氯乙酰基吲哚酮为原料, 与取代硫脲在绝对乙 醇中成功合成了一系列 5-(2-取代氨基噻唑-4-基)二氢吲 哚-2-酮类化合物. 为了证明实验路线及反应溶剂的实 用性, 我们采用同样的方法尝试 5-氯乙酰基吲哚酮与硫 代酰胺反应. 已有文献报道 ${ }^{[24]}$ 以 5-氯乙酰基吲哚酮为原 料, 与甲基硫代酰胺反应合成 5-(2-噻唑-4-基)二氢吲哚2-酮, 与乙基硫代酰胺反应合成 5-(2-甲基噻唑-4-基)二 氢吲哚-2-酮. 在文献基础上我们采用绝对乙醇溶剂, 考 察了底物与甲(乙)基硫代酰胺, 苯基和咔唑硫代酰胺的 反应, 合成了未见文献报道的 5-(2-取代噻唑-4-基)二氢 吲哚-2-酮(2l２q). 合成路线见图 3, 反应结果见表 3.<smiles>O=C1Cc2cc(C(=O)CCl)ccc2N1</smiles>

1

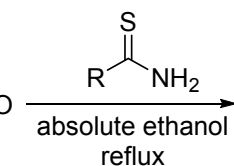

$\mathrm{CH}_{3} \mathrm{COONa}$<smiles>[R]c1nc(-c2ccc3c(c2)CC(=O)N3)cs1</smiles>

$2 \mathbf{q} \sim 2 \mathbf{q}$
图式 3 5-(2-取代噻唑-4-基)二氢吲哚-2-酮类化合物的合成 Scheme 3 Synthetic route of 5-(2-substituted-thiazol-4-yl)indolin-2-one derivatives

从反应结果可以看出在绝对乙醇溶剂中底物和取 代硫代酰胺缩合反应表现出较高的反应选择性和反应 活性, 文献合成 5-(2-噻唑-4-基)二氢吲哚-2-酮时采用二 啞烷和三乙胺为混合溶剂, 前后两次加入原料, 操作复 杂, 收率为 35\%; 合成 5-(2-甲基噻唑-4-基)二氢吲哚-2酮时采用乙酸为溶剂; 此外文献中仅合成了 5-(2-噻唑-

表 3 5-氯乙酰基吲哚酮与硫代酰胺反应结果

Table 3 Reaction results of 5-chloroacetyloxindole and thioamide

\begin{tabular}{|c|c|c|c|c|}
\hline Entry & Compd. & m.p. $/{ }^{\circ} \mathrm{C}$ & Time/h & Yield $^{a} / \%$ \\
\hline 1 & 21 & $242.1 \sim 243.5$ & 7 & 67 \\
\hline 2 & $2 m$ & $263.0 \sim 264.8$ & 8 & 88 \\
\hline 3 & $2 n$ & $234.5 \sim 234.8$ & 8 & 83 \\
\hline 4 & 20 & $>300$ & 11 & 78 \\
\hline 5 & 20 & $>300$ & 13 & 81 \\
\hline 6 & $2 q$ & $>300$ & 13 & 80 \\
\hline
\end{tabular}

${ }^{a}$ 分离产率. 
4-基)二氢吲哚-2-酮和 5-(2-甲基噻唑-4-基)二氢吲哚-2酮. 我们选用绝对乙醇为溶剂更为安全, 具有适用性, 同时所合成的化合物产率均在 $67 \% \sim 88 \%$ 之间.

\section{3 结构表征}

为了证实结构，以 5-[2-(2-甲苯基氨基)噻唑-4-基] 二氢吲哚-2-酮(2b)为例说明. ${ }^{1} \mathrm{H}$ NMR 谱图: $\delta 10.47$ 处出 现单峰, 归属于吲哚环 $\mathrm{N}$ 上氢质子吸收峰; $\delta 9.30$ 处出 现单峰, 归属于氨基 $\mathrm{N}$ 上氢质子吸收峰; $\delta 8.01 \sim 7.01$ 处 出现吸收峰, 归属于苯环上氢质子吸收峰; $\delta 6.85$ 处出 现单峰, 归属于噻唑环上氢质子吸收峰; $\delta 3.53$ 处出现 单峰，归属于吲哚酮环亚甲基上氢质子吸收峰; $\delta 2.29$ 处出现单峰, 归属于苯环上甲基氢质子吸收峰. 该产物 的 ${ }^{13} \mathrm{C}$ NMR 结构中, $\delta 176.8$ 处出现吲哚环羰基碳的吸收 峰, $\delta 36.25$ 处出现吲哚环上亚甲基碳吸收峰, $\delta 18.47$ 处 出现苯环上甲基碳吸收峰. 该在 IR 图中我们发现在 $1647 \mathrm{~cm}^{-1}$ 处出现吲哚环羰基的伸缩振动峰, 在 1715 , $1687,1232,1123 \mathrm{~cm}^{-1}$ 处出现噻唑环伸缩振动峰. 该产物 的元素分析数据也与我们推测的化合物结构相符. 以上 数据证实了得到的产物为 5-[2-(2-甲苯基氨基)噻唑-4基]二氢吲哚-2-酮(2b). 其他产物的结构也经 ${ }^{1} \mathrm{H}$ NMR, ${ }^{13} \mathrm{C} N M R$, IR 和元素分析得以证实.

\section{2 结论}

5-氯乙酰基吲哚酮(1)为原料, 与硫䐂(取代硫艮)、 硫代酰胺在绝对乙醇为溶剂的条件下，以 $67 \% \sim 89 \%$ 的 收率成功地合成了一系列具有潜在生物活性的噻唑联 吲哚类化合物 $\mathbf{2 a} \sim 2$ q. 为构建新型的噻唑环修饰的吲 哚类化合物提供了有效的合成策略, 同时为寻找新型生 理活性药物提供先导化合物以及实验依据. 该方法具有 操作简单、反应条件温和、后处理方便等优点, 有较好 的应用前景.

\section{3 实验部分}

\section{1 仪器与试剂}

熔点采用 WRS-1B 数字熔点仪测定(温度计未校 正); IR 采用 Nicolet AVAR 360 FT-IR 型傅里叶变换红外 分光光度计测定; 核磁共振氢谱采用 Agilent 1100- 400 $\mathrm{MHz}$ 型核磁共振仪测定, DMSO- $d_{6}$ 为溶剂, TMS 为内 标; 质谱采用 Agilent 1100 质谱仪测定; 元素分析由 Elementar Vario EL 测定. 所用的药品均为市售分析纯 或化学纯或按文献方法制备.

\section{2 实验方法}

\subsection{1 氯乙酰基吲哚酮的合成}

将氯乙酰氯 $5.08 \mathrm{~g}(45 \mathrm{mmol})$ 和三氯化铝 $6.00 \mathrm{~g}$ (45 $\mathrm{mmol}$ )的二氯甲烷溶液在搅拌的条件下, 逐滴加入含有 吲哚酮 $4.0 \mathrm{~g}(30 \mathrm{mmol})$ 的二氯甲烷 $(30 \mathrm{~mL})$ 溶液中, 冰盐 浴反应 $2 \mathrm{~h}$. 反应完毕后加水, 继续反应 $1 \mathrm{~h}$, 蒸除溶剂, 抽滤, 所得粗产物经冰乙酸重结晶得到产物 1 . 收率为 $78 \%$, m.p. $121.3 \sim 122.4{ }^{\circ} \mathrm{C}$ (文献值 ${ }^{[24]}$ : 产率 53\%, m.p. $\left.123.4 \sim 124.1{ }^{\circ} \mathrm{C}\right)$.

\subsection{2 吲哚酮联噻唑化合物的合成}

氯乙酰基吲哚酮(1) (1 mmol, $0.21 \mathrm{~g}$ )和硫嫝(取代硫 嘱)或硫代酰胺 $(1 \mathrm{mmol})$ 加入绝对乙醇 $(6 \mathrm{~mL})$ 溶剂中, 回 流反应, 用 TLC 进行监测. 反应完全后, 用饱和乙酸钠 溶液调节 $\mathrm{pH}$ 至中性, 抽滤, 洗涤, 粗产物用冰醋酸和乙 酸乙酯混合溶剂重结晶得到产物 $\mathbf{2 a} \sim \mathbf{2 q}$.

5-(2-氨基噻唑-4-基)二氢吲哚-2-酮(2a)：白色粉末, 产率 82\%. m.p. 278.9 280.2 ${ }^{\circ} \mathrm{C}$; ${ }^{1} \mathrm{H}$ NMR $(400 \mathrm{MHz}$, DMSO- $\left.d_{6}\right) \delta: 10.41(\mathrm{~s}, 1 \mathrm{H}, \mathrm{NH}), 7.61(\mathrm{~s}, 2 \mathrm{H}, \mathrm{ArH}), 6.98$ (s, $2 \mathrm{H}, \mathrm{ArH}, \mathrm{CH}), 6.77$ (s, $\left.2 \mathrm{H}, \mathrm{NH}_{2}\right), 3.47$ (s, $\left.2 \mathrm{H}, \mathrm{CH}_{2}\right) ;{ }^{13} \mathrm{C}$ NMR $\left(100 \mathrm{MHz}\right.$, DMSO- $\left.d_{6}\right) \delta: 176.90,168.40,150.45$, 143.29, 129.07, 126.44, 125.49, 122.24, 109.35, 99.57, 36.24; IR (KBr) v: 3420, 3291, 2982, 1735, 1665, 1230, $1124 \mathrm{~cm}^{-1}$; ESI-MS $m / z$ : $231.0[\mathrm{M}+\mathrm{H}]^{+}$. Anal. calcd for $\mathrm{C}_{11} \mathrm{H}_{9} \mathrm{~N}_{3} \mathrm{OS}$ : C 57.13, H 3.92, N 18.17; found C 57.16, H 3.93, N 18.15 .

5-[2-(苯基氨基)噻唑-4-基]二氢吲哚-2-酮 (2b): 灰 色固体, 产率 $89 \%$. m.p. $240.8 \sim 241.6{ }^{\circ} \mathrm{C} ;{ }^{1} \mathrm{H}$ NMR (400 MHz, DMSO- $\left.d_{6}\right) \delta: 10.49$ (s, 1H, NH), $10.25(\mathrm{~s}, 1 \mathrm{H}, \mathrm{NH})$, 7.77 (s, 2H, ArH), 7.72 (d, J=7.0 Hz, 2H, ArH), 7.35 (s, 2H, ArH), 7.16 (s, 1H, ArH), 6.96 (s, 1H, ArH), 6.87 (d, $J=7.4 \mathrm{~Hz}, 1 \mathrm{H}, \mathrm{CH}), 3.56\left(\mathrm{~s}, 2 \mathrm{H}, \mathrm{CH}_{2}\right) ;{ }^{13} \mathrm{C}$ NMR $(100$ MHz, DMSO- $\left.d_{6}\right) \delta$ : $176.91,163.28,150.77,143.65$, $141.65,129.44,128.65,126.65,125.66,122.29,121.53$, 117.13, 109.52, 100.98, 36.27; IR (KBr) v: 3446, 3235, 2972, 1725, 1675, 1233, $1104 \mathrm{~cm}^{-1}$; ESI-MS m/z: 307.1 $[\mathrm{M}+\mathrm{H}]^{+}$. Anal. calcd for $\mathrm{C}_{17} \mathrm{H}_{13} \mathrm{~N}_{3} \mathrm{OS}: \mathrm{C} 66.43, \mathrm{H} 4.26, \mathrm{~N}$ 13.67; found C 66.50, H 4.31, N 13.60.

5-[2-(2-甲苯基氨基)噻唑-4-基]二氢吲哚-2-酮(2c): 黄色晶体, 产率 86\%. m.p. 257.6 258.3 ${ }^{\circ} \mathrm{C} ;{ }^{1} \mathrm{H}$ NMR $\left(400 \mathrm{MHz}, \mathrm{DMSO}-d_{6}\right) \delta: 10.48$ (s, $\left.1 \mathrm{H}, \mathrm{NH}\right), 9.30(\mathrm{~s}, 1 \mathrm{H}$, $\mathrm{NH}), 8.01(\mathrm{~s}, 1 \mathrm{H}, \mathrm{ArH}), 7.71(\mathrm{~s}, 2 \mathrm{H}, \mathrm{ArH}), 7.23$ (s, 2H, ArH), 7.08 (s, 1H, ArH), 7.01 (s, 1H, ArH), 6.85 (s, 1H, $\mathrm{CH}$ ), 3.53 (s, $2 \mathrm{H}, \mathrm{CH}_{2}$ ), 2.29 (s, 3H, $\left.\mathrm{CHR}_{3} \mathrm{R}\right) ;{ }^{13} \mathrm{C} \mathrm{NMR}$ $\left(100 \mathrm{MHz}, \mathrm{DMSO}-d_{6}\right) \delta: 176.89,165.48,150.56,143.55$, $139.88,131.05,129.11,128.78,126.97,126.58,125.62$, 123.66, 122.23, 121.25, 109.44, 101.02, 36.25, 18.47; IR (KBr) v: 3445, 3230, 2953, 1715, 1687, 1232, $1123 \mathrm{~cm}^{-1}$; ESI-MS $m / z$ : $321.1[\mathrm{M}+\mathrm{H}]^{+}$. Anal. calcd for $\mathrm{C}_{18} \mathrm{H}_{15} \mathrm{~N}_{3} \mathrm{OS}$ : 
C 67.27, H 4.70, N 13.07; found C 67.30, H 4.71, N 13.04. 5-[2-(3-甲苯基氨基)噻唑-4-基]二氢吲哚-2-酮(2d): 白色晶体, 产率 $80 \%$. m.p. 232.0 233.8 ${ }^{\circ} \mathrm{C} ;{ }^{1} \mathrm{H} \mathrm{NMR}$ (400 MHz, DMSO- $\left.d_{6}\right) \delta: 10.50$ (s, 1H, NH), 10.18 (s, 1H, NH), 7.77 (d, $J=7.7 \mathrm{~Hz}, 2 \mathrm{H}, \operatorname{ArH}), 7.61$ (d, $J=7.9 \mathrm{~Hz}$, 1H, ArH), 7.44 (s, 1H, ArH), 7.23 (t, J=7.8 Hz, 1H, ArH), 7.14 (s, 1H, ArH), 6.88 (d, J=8.0 Hz, 1H, ArH), 6.79 (d, $J=7.3 \mathrm{~Hz}, 1 \mathrm{H}, \mathrm{CH}), 3.56\left(\mathrm{~s}, 2 \mathrm{H}, \mathrm{CH}_{2}\right), 2.32\left(\mathrm{~s}, 3 \mathrm{H}, \mathrm{CH}_{3}\right)$; ${ }^{13} \mathrm{C}$ NMR (100 MHz, DMSO- $\left.d_{6}\right) \quad \delta: 176.91,163.36$, $150.74,143.64,141.61,138.53,129.34,128.67,126.65$, $125.68,122.37,117.80,114.38,109.53,100.88,36.27$, 21.85; IR (KBr) v: 3440, 3226, 2955, 1718, 1685, 1234, $1120 \mathrm{~cm}^{-1}$; ESI-MS m/z: $321.1[\mathrm{M}+\mathrm{H}]^{+}$. Anal. calcd for $\mathrm{C}_{18} \mathrm{H}_{15} \mathrm{~N}_{3} \mathrm{OS}$ : C 67.27, H 4.70, N 13.07; found C 67.30, H 4.71, N 13.04.

5-[2-(2-乙基苯基氨基)噻唑-4-基]二氢吲哚-2-酤 (2e): 白色晶体, 产率 $84 \%$. m.p. $252.3 \sim 253.3{ }^{\circ} \mathrm{C}$; ${ }^{1} \mathrm{H}$ NMR (400 MHz, DMSO- $\left.d_{6}\right) \delta: 10.46$ (s, 1H, NH), 9.30 (s, 1H, NH), 7.90 (d, J=7.7 Hz, 1H, ArH), 7.69 (s, 2H, ArH), 7.24 (t, $J=8.2 \mathrm{~Hz}, 2 \mathrm{H}, \operatorname{ArH}), 7.06$ (d, $J=11.8 \mathrm{~Hz}, 2 \mathrm{H}$, ArH), 6.83 (d, J=8.3 Hz, 1H, CH), 3.52 (s, 2H, $\left.\mathrm{CH}_{2}\right), 2.69$ (dd, $\left.J=14.6,7.2 \mathrm{~Hz}, 2 \mathrm{H}, \mathrm{CH}_{2}\right), 1.14$ (t, $J=7.3 \mathrm{~Hz}, 3 \mathrm{H}$, $\left.\mathrm{CH}_{3}\right) ;{ }^{13} \mathrm{C}$ NMR (100 MHz, DMSO- $\left.d_{6}\right) \delta: 175.07,164.47$, $148.81,141.71,137.41,134.05,127.55,126.99,125.15$, $124.73,123.75,122.65,120.86,120.43,107.61,98.95$, 34.43, 22.42, 13.03; IR (KBr) v: 3436, 3230, 2956, 1719, 1683, 1231, $1120 \mathrm{~cm}^{-1}$; ESI-MS m/z: $335.1[\mathrm{M}+\mathrm{H}]^{+}$. Anal. calcd for $\mathrm{C}_{19} \mathrm{H}_{17} \mathrm{~N}_{3} \mathrm{OS}$ : C 68.03, H 5.11, N 12.53; found $\mathrm{C} 68.06, \mathrm{H} 5.13, \mathrm{~N} 12.50$.

5-\{2-[(4-甲氧基苯基)氨基]噻唑-4-基 $\}$ 二氢吲哚-2酮(2f): 棕色晶体, 产率 84\%. m.p. $276.5 \sim 276.9{ }^{\circ} \mathrm{C} ;{ }^{1} \mathrm{H}$ NMR (400 MHz, DMSO-d $)) \delta: 10.47$ (s, 1H, NH), 10.02 (s, 1H, NH), 7.75 (s, 2H, ArH), 7.63 (d, J=8.7 Hz, 2H, ArH), 7.08 (s, 1H, ArH), 6.94 (d, J=8.7 Hz, 2H, ArH), $6.86(\mathrm{~d}, J=8.5 \mathrm{~Hz}, 1 \mathrm{H}, \mathrm{CH}), 3.74\left(\mathrm{~s}, 3 \mathrm{H}, \mathrm{CH}_{3}\right), 3.55$ (s, $\left.2 \mathrm{H}, \mathrm{CH}_{2}\right) ;{ }^{13} \mathrm{C}$ NMR $\left(100 \mathrm{MHz}, \mathrm{DMSO}-d_{6}\right) \delta: 176.36$, $163.37,153.86,150.16,143.04,134.65,128.20,126.06$, $125.07,121.75,118.38,114.11,108.93,99.71,55.07$, 35.73; IR (KBr) v: 3445, 3233, 2952, 1717, 1683, 1236, $1124 \mathrm{~cm}^{-1}$; ESI-MS m/z: $337.1[\mathrm{M}+\mathrm{H}]^{+}$. Anal. calcd for $\mathrm{C}_{18} \mathrm{H}_{15} \mathrm{~N}_{3} \mathrm{O}_{2} \mathrm{~S}$ : C 68.08, H 4.48, N 12.43; found C 68.11, H 4.51, N 12.40 .

5-\{2-[(2-羟基苯基)氨基]噻唑-4-基 $\}$ 二氢吲哚-2-酮 (2g): 灰色粉末, 产率 $83 \%$. m.p. 267.5 268.1 ${ }^{\circ} \mathrm{C} ;{ }^{1} \mathrm{H}$ NMR (400 MHz, DMSO- $\left.d_{6}\right) \delta$ : 10.47 (s, 1H, NH), 9.95 (s,
$1 \mathrm{H}, \mathrm{OH}), 9.45$ (s, 1H, NH), $8.32 \sim 8.20$ (s, 1H, ArH), 7.73 (s, 2H, ArH), 7.09 (s, 1H, ArH), 6.87 (d, J=7.9 Hz, 1H, $\mathrm{CH}), 3.55\left(\mathrm{~s}, 2 \mathrm{H}, \mathrm{CH}_{2}\right) ;{ }^{13} \mathrm{C}$ NMR (100 MHz, DMSO- $\left.d_{6}\right)$ $\delta: 176.90,164.35,150.15,146.68,143.54,129.74,128.72$, $126.63,125.52,122.66,122.20,119.69,119.29,115.47$, 109.51, 101.33, 36.27; IR (KBr) v: 3374, 3271, 2987, 1668, 1621, 1261, $1119 \mathrm{~cm}^{-1}$; ESI-MS m/z: $323.1 \mathrm{M}+$ $\mathrm{H}]^{+}$. Anal. calcd for $\mathrm{C}_{17} \mathrm{H}_{13} \mathrm{~N}_{3} \mathrm{OR}_{2} \mathrm{~S}$ : C 63.14, $\mathrm{H} 4.05, \mathrm{~N}$ 12.99; found $\mathrm{C} 63.16, \mathrm{H} 4.06, \mathrm{~N} 12.98$.

5-\{2-[(4-硝基苯基)氨基]噻唑-4-基 $\}$ 二氢吲哚-2-酮 (2h): 黄色晶体, 产率 74\%. m.p. $>300{ }^{\circ} \mathrm{C} ;{ }^{1} \mathrm{H}$ NMR (400 MHz, DMSO- $\left.d_{6}\right) \delta: 11.07$ (s, 1H, NH), $10.51(\mathrm{~s}, 1 \mathrm{H}, \mathrm{NH})$, $8.27(\mathrm{~d}, J=8.6 \mathrm{~Hz}, 2 \mathrm{H}, \mathrm{ArH}), 7.94$ (d, $J=8.8 \mathrm{~Hz}, 2 \mathrm{H}$, ArH), 7.83 (d, J=7.6 Hz, 2H, ArH), 7.37 (s, 1H, ArH), $6.89(\mathrm{~d}, J=8.0 \mathrm{~Hz}, 1 \mathrm{H}, \mathrm{CH}), 3.57\left(\mathrm{~s}, 2 \mathrm{H}, \mathrm{CH}_{2}\right) ;{ }^{13} \mathrm{C} \mathrm{NMR}$ $\left(100 \mathrm{MHz}, \mathrm{DMSO}-d_{6}\right) \delta: 176.89,170.78,161.91,151.09$, $147.32,143.92,140.52,128.19,126.74,126.03,122.43$, 116.57, 109.56, 103.35, 36.28; IR (KBr) v: 3445, 3233, 2952, 1717, 1683, 1236, $1124 \mathrm{~cm}^{-1}$; ESI-MS m/z: 352.1 $[\mathrm{M}+\mathrm{H}]^{+}$. Anal. calcd for $\mathrm{C}_{17} \mathrm{HR}_{12} \mathrm{~N}_{4} \mathrm{O}_{3} \mathrm{~S}$ : C 57.95, H 3.43, N 15.90; found C 57.96, H 3.44, N 15.89.

5-\{2-[(4-溴苯基)氨基]噻唑-4-基 $\}$ 二氢吲哚-2-酮 (2i): 黄色晶体，产率 $80 \%$. m.p. 266.5 267.8 ${ }^{\circ} \mathrm{C} ;{ }^{1} \mathrm{H}$ NMR (400 MHz, DMSO-d $) \delta: 10.48$ (s, 1H, NH), 10.39 (s, 1H, NH), 7.76 (d, $J=6.4 \mathrm{~Hz}, 2 \mathrm{H}, \mathrm{ArH}), 7.71$ (d, $J=8.6$ $\mathrm{Hz}, 2 \mathrm{H}, \mathrm{ArH}), 7.51$ (d, J=8.4 Hz, 2H, ArH), 7.20 (s, 1H, ArH), 6.87 (d, $J=8.5 \mathrm{~Hz}, 1 \mathrm{H}, \mathrm{CH}), 3.55\left(\mathrm{~s}, 2 \mathrm{H}, \mathrm{CH}_{2}\right) ;{ }^{13} \mathrm{C}$ NMR (100 MHz, DMSO- $\left.d_{6}\right) \delta: 176.89,162.89,150.81$, $143.72,140.92,132.12,128.50,126.66,125.71,122.34$, 119.09, 112.61, 109.50, 101.48, 36.28; IR (KBr) v: 3374, 3271, 3168, 1698, 1621, 1261, $1119 \mathrm{~cm}^{-1}$; ESI-MS m/z: $385.0[\mathrm{M}+\mathrm{H}]^{+}$. Anal. calcd for $\mathrm{C}_{17} \mathrm{H}_{12} \mathrm{BrN}_{3} \mathrm{OS}$ : C 52.86, $\mathrm{H} 3.13, \mathrm{~N} 10.88$; found C 52.86, H 3.14, N 10.87 .

5-[2-(2-䒬基氨基)噻唑-4-基]二氢吲哚-2-酮 $(\mathbf{2} \mathbf{j})$ : 白 色粉末, 产率 $77 \%$. m.p. $290.5 \sim 291.4{ }^{\circ} \mathrm{C} ;{ }^{1} \mathrm{H}$ NMR (400 MHz, DMSO- $\left.d_{6}\right) \delta: 10.54(\mathrm{~s}, 1 \mathrm{H}, \mathrm{NH}), 10.18(\mathrm{~s}, 1 \mathrm{H}, \mathrm{NH})$, 8.39 (t, $J=7.7 \mathrm{~Hz}, 2 \mathrm{H}, \operatorname{ArH}), 8.00$ (d, $J=7.2 \mathrm{~Hz}, 1 \mathrm{H}$, ArH), 7.81 (s, 2H, ArH), 7.71 (d, $J=8.0 \mathrm{~Hz}, 1 \mathrm{H}, \operatorname{ArH})$, $7.60(\mathrm{t}, J=7.3 \mathrm{~Hz}, 3 \mathrm{H}, \operatorname{ArH}), 7.21$ (s, 1H, ArH), $6.91(\mathrm{~s}$, $J=8.1 \mathrm{~Hz}, 1 \mathrm{H}, \mathrm{NH}), 3.60\left(\mathrm{~s}, 2 \mathrm{H}, \mathrm{CH}_{2}\right) ;{ }^{13} \mathrm{C} \mathrm{NMR}(100$ $\left.\mathrm{MHz}, \mathrm{DMSO}-d_{6}\right) \delta: 175.08,163.55,148.79,141.80$, 135.29 , 132.56, 126.90, 124.54, 123.84, 121.56, 120.90, 114.55, 107.69, 99.78, 34.45; IR (KBr) v: 3446, 3279, 3071, 1686, 1602, 1236, $1120 \mathrm{~cm}^{-1}$; ESI-MS m/z: 357.1 $[\mathrm{M}+\mathrm{H}]^{+}$. Anal. calcd for $\mathrm{C}_{21} \mathrm{H}_{15} \mathrm{~N}_{3} \mathrm{OS}$ : C 70.57, $\mathrm{H} 4.23, \mathrm{~N}$ 
11.76; found C 70.58, H 4.23, N 11.75.

5-[2-(苯并 $[d][1,3]$ 二氧杂环戊烯-5-基氨基)噻唑-4基]二氢吲哚-2-酮(2k): 白色粉末, 产率 79\%. m.p. $282.5 \sim 283.7{ }^{\circ} \mathrm{C} ;{ }^{1} \mathrm{H}$ NMR (400 MHz, DMSO- $d_{6}$ ) $\delta: 10.48$ (s, 1H, NH), 10.12 (s, 1H, NH), 7.74 (s, 2H, ArH), 7.51 (s, $1 \mathrm{H}, \operatorname{ArH}), 7.11$ (d, $J=1.6 \mathrm{~Hz}, 1 \mathrm{H}, \operatorname{ArH}), 7.09 \sim 7.02(\mathrm{~m}$, $1 \mathrm{H}, \mathrm{ArH}), 6.01\left(\mathrm{~s}, 2 \mathrm{H}, \mathrm{CH}_{2}\right), 3.56\left(\mathrm{~s}, 2 \mathrm{H}, \mathrm{CH}_{2}\right) ;{ }^{13} \mathrm{C} \mathrm{NMR}$ $\left(100 \mathrm{MHz}, \mathrm{DMSO}-d_{6}\right) \delta: 176.90,163.68,150.72,147.77$, $143.65,141.95,136.53,128.69,126.65,125.60,122.26$, $109.87,109.52,108.80,101.27,100.58,99.83,36.27$; IR (KBr) v: 3445, 3280, 3075, 1685, 1604, 1236, $1133 \mathrm{~cm}^{-1}$; ESI-MS $m / z$ : $351.1[\mathrm{M}+\mathrm{H}]^{+}$. Anal. calcd for $\mathrm{C}_{18} \mathrm{H}_{13} \mathrm{~N}_{3}-$ $\mathrm{O}_{3} \mathrm{~S}$ : C 61.53, H 3.73, N 11.96; found C 61.55, H 3.74, N 11.95 .

5-(2-噻唑-4-基)二氢吲哚-2-酮(2I): 白色粉末, 产率 67\%. m.p. 242.1 243.5 ${ }^{\circ} \mathrm{C} ;{ }^{1} \mathrm{H}$ NMR (400 MHz, DMSO$\left.d_{6}\right) \delta: 10.47(\mathrm{~s}, 1 \mathrm{H}, \mathrm{NH}), 9.14(\mathrm{~s}, 1 \mathrm{H}, \mathrm{CH}), 7.97(\mathrm{~s}, 1 \mathrm{H}$, ArH), $7.85 \sim 7.70(\mathrm{~m}, 2 \mathrm{H}, \mathrm{ArH}), 6.87(\mathrm{~s}, 1 \mathrm{H}, \mathrm{CH}), 3.53(\mathrm{~s}$, $\left.2 \mathrm{H}, \mathrm{CH}_{2}\right) ;{ }^{13} \mathrm{C}$ NMR $\left(100 \mathrm{MHz}, \mathrm{DMSO}-d_{6}\right) \delta: 176.83$, $165.48,154.43,143.86,127.26,126.76,125.93,122.63$, 110.78, 109.53, 36.12; IR (KBr) v: 3422, 3146, 2955, 1722, 1623, 1471, 1236, $1155 \mathrm{~cm}^{-1}$; ESI-MS m/z: 216.0 $[\mathrm{M}+\mathrm{H}]^{+}$. Anal. calcd for $\mathrm{C}_{11} \mathrm{H}_{8} \mathrm{~N}_{2} \mathrm{OS}$ : C 61.09, H 3.73, N 12.95; found C 61.11, H 3.74, N 12.53 .

5-(2-甲基噻唑-4-基)二氢吲哚-2-酮 $(\mathbf{2 m})$ : 白色粉末, 产率 88\%. m.p. $263.0 \sim 264.8{ }^{\circ} \mathrm{C}$; ${ }^{1} \mathrm{H}$ NMR $(400 \mathrm{MHz}$, DMSO- $\left.d_{6}\right) \delta: 10.46(\mathrm{~s}, 1 \mathrm{H}, \mathrm{NH}), 7.80 \sim 7.68(\mathrm{~m}, 3 \mathrm{H}, \mathrm{ArH})$, $6.82(\mathrm{t}, J=6.1 \mathrm{~Hz}, 1 \mathrm{H}, \mathrm{CH}), 3.51\left(\mathrm{~s}, 2 \mathrm{H}, \mathrm{CH}_{2}\right), 2.66(\mathrm{~s}$, $\left.3 \mathrm{H}, \mathrm{CH}_{3}\right) ;{ }^{13} \mathrm{C} \mathrm{NMR}\left(100 \mathrm{MHz}, \mathrm{DMSO}-d_{6}\right) \delta: 176.88$, $165.58,154.48,143.89,128.26,126.76,125.93,122.63$, 111.78, 109.57, 36.22, 19.37; IR (KBr) v: 3420, 3145, 2965, 1732, 1633, 1475, 1228, $1156 \mathrm{~cm}^{-1}$; ESI-MS m/z: $230.0[\mathrm{M}+\mathrm{H}]^{+}$. Anal. calcd for $\mathrm{C}_{12} \mathrm{H}_{10} \mathrm{~N}_{2} \mathrm{OS}$ : C 62.59, H 4.38, N 12.16; found C 62.60, H 4.39, N 12.14.

5-(2-苯基噻唑-4-基)二氢吲哚-2-酮(2n): 白色粉末, 产率 83\%. m.p. 234.5 234.8 ${ }^{\circ} \mathrm{C}$; ${ }^{1} \mathrm{H}$ NMR (400 MHz, DMSO- $\left.d_{6}\right) \delta$ : $10.55(\mathrm{~s}, 1 \mathrm{H}, \mathrm{NH}), 8.02(\mathrm{~d}, J=9.8 \mathrm{~Hz}, 3 \mathrm{H}$, ArH), 7.91 (d, $J=7.4 \mathrm{~Hz}, 2 \mathrm{H}, \operatorname{ArH}), 7.53$ (d, $J=6.7 \mathrm{~Hz}$, $3 \mathrm{H}, \mathrm{ArH}), 6.91(\mathrm{~d}, J=8.3 \mathrm{~Hz}, 1 \mathrm{H}, \mathrm{CH}), 3.58$ (s, 2H, $\left.\mathrm{CHR}_{2} \mathrm{R}\right) ;{ }^{13} \mathrm{C} \mathrm{NMR}\left(100 \mathrm{MHz}, \mathrm{DMSO}-d_{6}\right) \delta: 176.93$, $167.04,155.89,144.22,133.48,130.72,129.68,127.99$, $126.86,126.58,126.11,122.84,112.82,109.65,36.24$; IR (KBr) v: 3422, 3146, 2955, 1722, 1623, 1471, 1236, 1155 $\mathrm{cm}^{-1}$; ESI-MS m/z: $292.1[\mathrm{M}+\mathrm{H}]^{+}$. Anal. calcd for $\mathrm{C}_{17} \mathrm{H}_{12} \mathrm{~N}_{2} \mathrm{OS}$ : C 69.84, H 4.14, N 9.58; found C 69.86, H

\subsection{4, N 9.57.}

5-[2-(9H-咔唑-3-基)噻唑-4-基]二氢吲哚-2-酮(20): 棕色粉末, 产率 78\%. m.p.>300 ${ }^{\circ} \mathrm{C} ;{ }^{1} \mathrm{H}$ NMR (400 MHz, DMSO- $\left.d_{6}\right) \delta: 11.60(\mathrm{~s}, 1 \mathrm{H}, \mathrm{NH}), 10.56(\mathrm{~s}, 1 \mathrm{H}, \mathrm{NH}), 8.80$ (s, 1H, ArH), 8.31 (d, $J=6.9 \mathrm{~Hz}, 1 \mathrm{H}, \operatorname{ArH}), 8.09$ (d, $J=7.8$ $\mathrm{Hz}, 1 \mathrm{H}, \mathrm{ArH}), 7.94$ (d, $J=16.8 \mathrm{~Hz}, 3 \mathrm{H}, \mathrm{ArH}), 7.61$ (d, $J=$ $8.0 \mathrm{~Hz}, 1 \mathrm{H}, \mathrm{ArH}), 7.55$ (d, J=7.7 Hz, 1H, ArH), 7.48 7.41 (m, 1H, ArH), 7.23 (s, 1H, ArH), 6.92 (s, 1H, CH), $3.60\left(\mathrm{~s}, 2 \mathrm{H}, \mathrm{CH}_{2}\right) ;{ }^{13} \mathrm{C} \mathrm{NMR}\left(100 \mathrm{MHz}, \mathrm{DMSO}-d_{6}\right) \delta$ : $175.13,166.76,153.69,142.26,139.48,138.94,126.48$, $124.93,124.29,122.88,121.39,120.96,119.39,117.80$, 116.94, 110.39, 107.80, 34.46; IR (KBr) v: 3511, 3341, 2985, 1688, 1615, 1443, $1117 \mathrm{~cm}^{-1}$; ESI-MS m/z: 381.1 $[\mathrm{M}+\mathrm{H}]^{+}$. Anal. calcd for $\mathrm{C}_{23} \mathrm{H}_{15} \mathrm{~N}_{3} \mathrm{OS}$ : C 72.42, H 3.96, N 11.02; found C 72.45, H 3.96, N 11.01.

5-[2-(9-甲基-9H-咔唑-3-基)噻唑-4-基]二氢吲哚-2酮(2p): 黄色粉末, 产率 $81 \%$. m.p.>300 ${ }^{\circ} \mathrm{C} ;{ }^{1} \mathrm{H} \mathrm{NMR}$ $\left(400 \mathrm{MHz}, \mathrm{DMSO}-d_{6}\right) \delta: 10.56(\mathrm{~s}, 1 \mathrm{H}, \mathrm{NH}), 8.82(\mathrm{~s}, 1 \mathrm{H}$, ArH), 8.34 (d, $J=7.5 \mathrm{~Hz}, 1 \mathrm{H}, \operatorname{ArH}), 8.15$ (d, $J=8.5 \mathrm{~Hz}$, 1H, ArH), 7.97 (s, 2H, ArH), 7.92 (s, 1H, ArH), 7.71 (d, $J=8.6 \mathrm{~Hz}, 1 \mathrm{H}, \mathrm{ArH}), 7.64(\mathrm{~d}, J=8.1 \mathrm{~Hz}, 1 \mathrm{H}, \mathrm{ArH})$, $7.55 \sim 7.50(\mathrm{~m}, 1 \mathrm{H}, \mathrm{ArH}), 7.28(\mathrm{~d}, J=14.8 \mathrm{~Hz}, 1 \mathrm{H}, \mathrm{ArH})$, $6.93(\mathrm{~d}, J=8.2 \mathrm{~Hz}, 1 \mathrm{H}, \mathrm{CH}), 3.92$ (s, 3H, $\left.\mathrm{CH}_{3}\right), 3.60$ (s, $\left.2 \mathrm{H}, \mathrm{CH}_{2}\right) ;{ }^{13} \mathrm{C}$ NMR (100 MHz, DMSO- $\left.d_{6}\right) \delta: 175.13$, $166.58,153.73,142.26,140.22,139.84,126.46,124.98$, $123.02,122.81,120.94,120.56,119.40,118.02,116.92$, 109.54, 107.98, 34.45, 27.84; IR (KBr) v: 3481, 3201, 2875, 1698, 1625, 1446, $1217 \mathrm{~cm}^{-1}$; ESI-MS m/z: 395.1 $[\mathrm{M}+\mathrm{H}]^{+}$. Anal. calcd for $\mathrm{C}_{24} \mathrm{H}_{17} \mathrm{~N}_{3} \mathrm{OS}$ : C 72.89, H 4.33, N 10.63; found C 72.90, H 4.33, N 10.62.

5-[2-(9-乙基-9H-咔唑-3-基)噻唑-4-基]二氢吲哚-2酮(2q): 白色粉末, 产率 $80 \%$. m.p. $>300{ }^{\circ} \mathrm{C} ;{ }^{1} \mathrm{H} \mathrm{NMR}$ $\left(400 \mathrm{MHz}, \mathrm{DMSO}-d_{6}\right) \delta: 10.59$ (s, 1H, NH), 8.84 (s, 1H, $\operatorname{ArH}), 8.35$ (d, $J=6.0 \mathrm{~Hz}, 1 \mathrm{H}, \operatorname{ArH}), 8.13(\mathrm{~d}, J=6.3 \mathrm{~Hz}$, 1H, ArH), 7.99 (s, 2H, ArH), 7.92 (d, $J=5.5 \mathrm{~Hz}, 1 \mathrm{H}, \mathrm{ArH})$, $7.71(\mathrm{~d}, J=7.1 \mathrm{~Hz}, 1 \mathrm{H}, \operatorname{ArH}), 7.65(\mathrm{~d}, J=6.5 \mathrm{~Hz}, 1 \mathrm{H}$, $\operatorname{ArH}), 7.52$ (d, $J=6.5 \mathrm{~Hz}, 1 \mathrm{H}, \operatorname{ArH}), 7.28$ (d, $J=6.2 \mathrm{~Hz}$, $1 \mathrm{H}, \mathrm{ArH}), 6.96$ (d, $J=4.7 \mathrm{~Hz}, 1 \mathrm{H}, \mathrm{CH}), 4.48$ (s, 2H, $\mathrm{CH}_{2}$ ), $3.61\left(\mathrm{~s}, 2 \mathrm{H}, \mathrm{CH}_{2}\right), 1.35\left(\mathrm{~d}, J=6.1 \mathrm{~Hz}, 3 \mathrm{H}, \mathrm{CH}_{3}\right) ;{ }^{13} \mathrm{C} \mathrm{NMR}$ $\left(100 \mathrm{MHz}, \mathrm{DMSO}-d_{6}\right) \delta: 175.13,166.59,153.75,142.28$, $139.16,138.76,126.48,124.99,124.35,122.95,121.07$, $120.76,119.54,118.01,117.03,109.61,108.00,35.79$, 34.46, 12.37; IR (KBr) v: 3522, 3241, 2975, 1702, 1624, 1471, 1236, $1155 \mathrm{~cm}^{-1}$; ESI-MS m/z: $409.1[\mathrm{M}+\mathrm{H}]^{+}$. Anal. calcd for $\mathrm{C}_{25} \mathrm{H}_{19} \mathrm{~N}_{3} \mathrm{OS}$ : C 73.32, H 4.68, N 10.26; 
found C 73.33, H 4.68, N 10.25.

辅助材料(Supporting Information) 目标产物 $\mathbf{2 a} \sim 2 \mathrm{2q}$ 的核磁谱图. 这些材料可以免费从本刊网站 (http://sioc-journal.cn/)上下载.

\section{References}

[1] Siddiqui, N.; Arshad, M. F.; Ahsan, W.; Alam, M. S. Int. J. Pharm. Sci. Drug Res. 2009, 1, 136.

[2] Cui, S. F.; Wang, Y.; Lv, J. S.; Damu, G. L. V.; Zhou, C. H. Sci. China Chem. 2012, 24, 1105 (in Chinese).

(崔胜峰, 王艳, 吕敬松, DAMU Guri L. V., 周成合, 中国科 学化学, 2012, 24, 1105.)

[3] Liaras, K.; Geronikaki, A.; Glamočlija, J.; Ćirić, A.; Soković, M. Bioorg. Med. Chem. 2011, 19, 3135.

[4] Mayhoub, A. S.; Khaliq, M.; Botting, C.; Li, Z.; Kuhn, R. J.; Cushman, M. Bioorg. Med. Chem. 2011, 19, 3845.

[5] Shi, H. B.; Zhang, S. J.; Ge, Q. F.; Guo, D. W.; Cai, C. M.; Hu, W. X. Bioorg. Med. Chem. Lett. 2010, 20, 6555.

[6] Chitra, S.; Paul, N.; Muthusubramanian, S.; Manisankar, P.; Yogeeswari, P.; Sriram, D. Eur. J. Med. Chem. 2011, 46, 4897.

[7] Shi, Y.; Zhou, C. H. Bioorg. Med. Chem. Lett. 2011, 21, 956.

[8] Thompson, M. J.; Louth, J. C,; Little, S. M.; Chen, B. Bioorg. Med. Chem. Lett. 2011, 21, 3644.

[9] Liu, Y. X.; Wei, D. G.; Zhu, Y. R.; Liu, S. H.; Zhang, Y. L.; Zhao, Q. Q.; Cai, B. L.; Li, Y. H.; Song, H. B.; Liu, Y.; Wang, Y.; Huang, R. Q.; Wang, Q. M. J. Agric. Food. Chem. 2008, 56, 204.

[10] Yu, H.; Shao, L.; Fang, J. J. Organomet. Chem. 2007, 692, 991.
[11] Wang, D. L.; Han, S.; Huang, X. D.; Gu, Z. Chin. J. Org. Chem. 2009, 29, 1659 (in Chinese).

(王道林, 韩珊, 黄孝东, 谷峥, 有机化学, 2009, 29, 1659.)

[12] Yang, P.; Weng, J. Q.; Tan, C. X.; Wang, X. L. Chin. J. Org. Chem. 2009, 29, 2000 (in Chinese). (杨鹏, 翁建全, 谭成侠, 王秀莲, 有机化学, 2009, 29, 2000.)

[13] Zhou, Z. Z.; Chen, Q.; Yang, G. F. Chin. J. Org. Chem. 2008, 28, 1385 (in Chinese). (周中振, 陈琼, 杨光富, 有机化学, 2008, 28, 1385.)

[14] Kutsunugia, Y.; Coudret, C.; Micheau, J. C.; Kawaia, T. Dyes Pigm. 2012, 92, 838.

[15] Zhang, J.; Li, Y.; Hui, C.; Zhu, J. Synth. Met. 2011, 161, 1166.

[16] Politanskaya, V.; Chuikov, I. P.; Shteingarts, V. D. Tetrahedron 2013, 69, 8477.

[17] Gao, W. T.; Tao, X. Y.; Lin, G. H.; Li, Y. Chin. J. Org. Chem. 2012, 32, 2171 (in Chinese). (高文涛, 陶希月, 林贵海, 李阳, 有机化学, 2012, 32, 2171.)

[18] Gao, W. T.; Lan, S.; Bairenqing, Z. M.; Li, Y. Chin. J. Org. Chem. 2014, 34, 2106 (in Chinese). (高文涛, 兰帅, 白仁青卓玛, 李阳, 有机化学, 2014, 34, 2106.)

[19] Hantzsch, A.; Weber, J. H. Ber. Dtsch. Chem. Ges. 1887, 20, 3118.

[20] Narender, M.; Somi, R. M.; Sridhar, R. Tetrahedron Lett. 2005, 46, 5953.

[21] Das, B.; Saidi, R. V.; Ramu, R. J. Mol. Catal. A: Chem. 2006, 252, 235.

[22] Rostamizadeh, S.; Aryan, R.; Ghaieni, H. R. Monatsh. Chem. 2008, 139, 1241.

[23] Jawale, D. V.; Lingampalle, D. V.; Pratap, U. R. Chin. Chem. Lett. 2010, 21, 412 .

[24] Berrey, S.; Hull, B. S.; Nelev, M.; Xue, Y. WO 2003/082853, 2003 [Chem. Abstr. 2003, 139, 323431].

(Li, L.; Fan, Y.) 